\title{
Semisynthesis of $\mathrm{NaK}$; a $\mathrm{Na}^{+}$and $\mathrm{K}+$ conducting ion channel
}

\author{
Kellie M. Linn $\ddagger$, Mehebaw G. Derebe§, Youxing Jiang§, and Francis I. Valiyaveetil ${ }^{\ddagger}{ }^{*}$ \\ ¥The Program in Chemical Biology, Department of Physiology and Pharmacology, Oregon Health \\ \& Science University, 3181 SW Sam Jackson Park Road, Portland, OR 97239 \\ $\S$ Department of Physiology, University of Texas Southwestern Medical Center, 5323 Harry Hines \\ Boulevard, Dallas, TX 75390
}

\begin{abstract}
In this contribution, we describe the semisynthesis of $\mathrm{NaK}$, a bacterial non-selective cation channel. In the semisynthesis, the $\mathrm{NaK}$ polypeptide is assembled from a recombinantly expressed thioester peptide and a chemically synthesized peptide using the native chemical ligation reaction. We describe a temporary tagging strategy for the purification of the hydrophobic synthetic peptide and demonstrate the efficient ligation of the synthetic peptide with the recombinant peptide thioester to form the semisynthetic NaK polypeptide. Following assembly, the NaK polypeptide is folded in vitro to the native state using lipid vesicles. Functional characterization of the folded semisynthetic $\mathrm{NaK}$ channels indicates that it is functionally similar to the wild type protein. We used semisynthesis to substitute aspartate 66 in the selectivity filter region of the $\mathrm{NaK}$ channel with the unnatural amino acids, homoserine and cysteine sulfonic acid. Functional analysis of these mutants suggests that the presence of a negatively charged residue in the vicinity of the ion binding sites is necessary for optimal flux of ions through the $\mathrm{NaK}$ channel.
\end{abstract}

Ion channels are integral membrane proteins that provide conduits for ions to pass through biological membranes.(1) One of the important functional characteristics of an ion channel is selectivity. Selectivity refers to the phenomenon by which channels permit the passage of only specific ions.(1) Ion channels display varying levels of selectivity that range from the high level of selectivity observed in $\mathrm{K}^{+}$channels, to the low level of selectivity observed in nonselective cation channels.(1) Non-selective cation channels, such as cyclic nucleotide gated ion channels (CNG channels), are selective for cations over anions but do not readily distinguish between different cations.(2)

The first structural view of a non-selective cation channel was provided by the high resolution structure of the bacterial ion channel, $\mathrm{NaK}$ (Fig. 1A).(3,4) The NaK channel, similar to CNG channels, conducts $\mathrm{Na}^{+}, \mathrm{K}^{+}, \mathrm{Rb}^{+}$and $\mathrm{Ca}^{2+}$ ions. $(3,5)$ The crystal structure shows that the overall structure of the $\mathrm{NaK}$ channel is very similar to the pore domain of $\mathrm{K}^{+}$channels like $\mathrm{KcsA}, \mathrm{K}_{\mathrm{v}} \mathrm{AP}$ or $\mathrm{K}_{\mathrm{v}}$ 1.2.(6-8) The major differences between the $\mathrm{NaK}$ channel and the $\mathrm{K}^{+}$ channels are in the ion binding regions, which are referred to as the "selectivity filters". A significant difference is that the selectivity filter of $\mathrm{K}^{+}$channels shows the presence of four ion binding sites while the selectivity filter in the $\mathrm{NaK}$ channel only shows the presence of two ion binding sites (Fig. 1B). $(3,4)$ The two ion binding sites in the NaK channel are structurally similar to the $3^{\text {rd }}$ and $4^{\text {th }}$ ion binding sites in the selectivity filter of a $\mathrm{K}^{+}$channel.(4) Even though the ion binding sites in the $\mathrm{NaK}$ channel and the $\mathrm{K}^{+}$channels are structurally similar, they exhibit different ion coordination properties.(4) Understanding the molecular basis for

*To whom correspondence should be addressed. Francis I. Valiyaveetil, Phone No: 503418 0946, Fax No: 5034944352 , valiyave@ ohsu.edu, Dept. of Physiology and Pharmacology, Mail code: L334, OHSU, Portland, OR 97239. 
this difference in ion coordination properties exhibited by structurally similar sites in the $\mathrm{NaK}$ channel and $\mathrm{K}^{+}$channels is of great scientific interest. However, these investigations will require the ability to manipulate the ion binding sites.

The ion binding sites in the $\mathrm{NaK}$ channel are built using the backbone carbonyl oxygen atoms of residues Thr63 and Val64 along with the side chain hydroxyl of Thr63 (Fig. 1B).(3,4) Due to the involvement of the protein backbone, traditional site directed mutagenesis methods can not be used to manipulate the ion binding sites. The protein backbone can be manipulated by chemical synthesis. Therefore, chemical synthesis of the NaK channel will allow us to manipulate the ion binding sites to understand details of its function.(9) In addition to modification of the protein backbone, chemical synthesis also allows the incorporation of a large number of unnatural amino acids, thereby providing an almost limitless variety of modifications that can be introduced into the NaK channel.(9) The ability to chemically synthesize the $\mathrm{NaK}$ channel will therefore be a great asset in structure-function investigations.

In spite of the advantages, chemical synthesis has been reported for only a few membrane proteins.(10) Chemical synthesis of membrane proteins is challenging due to the difficulties in the synthesis and purification of peptides that encompass transmembrane segments and the requirement for in vitro folding of the synthetic membrane protein. $(11,12)$ In this report, we describe the chemical synthesis of the NaK channel. We describe a semisynthetic approach in which the $\mathrm{NaK}$ channel is assembled from a recombinant peptide thioester and a synthetic peptide by native chemical ligation (NCL). Following assembly, we describe the in vitro folding of the $\mathrm{NaK}$ channel to the native tetrameric state and the functional characterization of the folded semisynthetic NaK channel. Further, we describe the use of semisynthesis to investigate Asp66 in the selectivity filter of the NaK channel. Our investigations demonstrate that the presence of a negatively charged residue at position 66 is required for optimal flux of ions through the $\mathrm{NaK}$ channel.

\section{Experimental Procedures}

\section{Chemical Synthesis of NaK 54-110 (C-Peptide)}

The peptide, $\mathrm{H}_{2} \mathrm{~N}$-CYFSVVTLTT VGDGNFSPQT DFGKIFTILY IFIGIGLVAG

FIHKLAVNVQ LPSILSG-COOH was synthesized by manual solid phase peptide synthesis (SPPS) (0.5 mmol scale) on a PAM resin using the in situ neutralization/2-(1H-benzo-triazol-1yl)-1,1,3,3-tetramethyluronium hexafluorophosphate (HBTU) activation protocol for Boc chemistry.(13) All the $\beta$-branched amino acids in the sequence were double-coupled using HBTU in dimethyl sulfoxide (DMSO).(14) After chain assembly, global de-protection and cleavage from the resin was achieved by treatment with anhydrous hydrogen fluoride (HF) containing $4 \% \mathrm{v} / \mathrm{v} p$-cresol, $1 \mathrm{~h}$ at $0{ }^{\circ} \mathrm{C}$. Following removal of HF, the crude peptide product was precipitated using cold anhydrous diethyl ether, washed thoroughly with diethyl ether containing 5\% $\beta$-mercaptoethanol, dissolved in 50\% trifluoroethanol (TFE): $\mathrm{H}_{2} \mathrm{O}$ containing $0.1 \%$ trifluoroacetic acid (TFA) and lyophilized.

The peptide used as a temporary solubilizing tag (TST, RRRC-CONH${ }_{2}$ ) was synthesized by manual SPPS (0.5 mmol scale) on a 4-methylbenzylhydrylamine (MBHA) resin using the in situ neutralization/HBTU activation protocol for Boc chemistry.(13) Following global deprotection and cleavage from the resin by anhydrous $\mathrm{HF}$, the crude peptide product was precipitated with diethyl ether, dissolved in 50\% Buffer B $\left(9: 1 \mathrm{MeCN} / \mathrm{H}_{2} \mathrm{O}, 0.1 \%\right.$ TFA) and lyophilized. The presence of the desired peptide in the crude was confirmed by reversed phase HPLC (RP-HPLC) and electrospray mass spectrometry (ES-MS) [observed mass $=589.2(\mathrm{M}$ $+\mathrm{H})$, expected $=589.7]$. 
For purification of the C-peptide, an aliquot of the C-peptide crude was dissolved in 50\% TFE: $\mathrm{H}_{2} \mathrm{O}, 0.3 \mathrm{M}$ HEPES, $\mathrm{pH}$ 8.0. An equal amount of the TST peptide was added and the mixture incubated at RT for 1 hour. The mixture was then acidified by addition of TFA and the C-peptide coupled to TST was purified by RP-HPLC on a preparative C4 column using a gradient of $60-100 \%$ buffer $\mathrm{C}$ (4.5:4.5:1 isopropanol/MeCN/ $\mathrm{H}_{2} \mathrm{O}, 0.1 \%$ TFA). Fractions containing the desired peptide were identified by ES-MS, pooled and lyophilized. The purified peptide was characterized as the desired product by ES-MS [observed mass $=6847.0 \pm 1$ (+dinitrophenol, DNP), expected $=6847.8]$.

The Asp residue (underlined) was replaced by homoserine (Hse) or cysteine sulfonic acid (Csa) to generate the Asp66 $\rightarrow$ Hse or the Asp66 $\rightarrow$ Csa substituted Cpeptides. The synthesis and purification of these peptides was carried out similar to the wild type peptide. For these peptides, removal of the DNP protecting group on the His residue was carried out using $\beta$ mercaptoethanol ( $20 \% \mathrm{v} / \mathrm{v})$ prior to HF cleavage. The purified peptides were characterized as the desired product by ES-MS [Asp66 $\rightarrow$ Hse C-peptide, observed mass $=6667.9 \pm 0.7$, expected $=6667.8 ;$ Asp66 $\rightarrow$ Csa C-peptide, observed mass $=6717.6 \pm 0.3$, expected $=6717.8]$.

\section{Expression and purification of the NaK 19-53 Thioester (N-Peptide)}

A dual fusion strategy was used for the expression of the N-peptide thioester.(14) The dual fusion consisted of NaK residues 19-53 sandwiched between glutathione-S-transferase (GST) at the $\mathrm{N}$-terminus and the gyrA intein-chitin binding domain at the C-terminus. A linker consisting of a thrombin site, His $_{6}$ and a Factor Xa site was introduced between GST and the $\mathrm{NaK}$ residues in the fusion. The fusion protein was expressed in Escherichia coli BL21(DE3) cells.

For purification, cells (from $1 \mathrm{~L}$ of culture) were resuspended in $50 \mathrm{~mL}$ of $50 \mathrm{mM}$ Tris- $\mathrm{HCl}$, pH 7.5, $0.2 \mathrm{M} \mathrm{NaCl}, 1 \mathrm{mM} \mathrm{MgCl}_{2}$, DNAse $(5 \mu \mathrm{g} / \mathrm{mL})$. Lysozyme $(0.1 \mathrm{mg} / \mathrm{mL})$ was added and the cells were incubated at room temperature with gentle stirring. After $30 \mathrm{~min}$, PMSF was added to $1 \mathrm{mM}$ and the cells were lysed by sonication, $5 \times 30 \mathrm{sec}$ bursts using a Branson model 450 sonifier at maximum power. After cell lysis, Triton X-100 was added to $1 \%(\mathrm{v} / \mathrm{v})$ and the cell lysate was incubated at room temperature for $30 \mathrm{~min}$. The soluble and insoluble fractions were separated by centrifugation at $12000 \mathrm{~g}$ for $10 \mathrm{~min}$ at room temperature. The insoluble fraction, which contains the fusion protein, was washed with $50 \mathrm{mM}$ Tris, $\mathrm{pH} 7.5,0.2 \mathrm{M} \mathrm{NaCl}$, $1 \%$ Triton $\mathrm{X}-100(\mathrm{v} / \mathrm{v}), 2 \mathrm{M}$ urea. The insoluble fraction obtained after the $2 \mathrm{M}$ urea wash was solubilized in $50 \mathrm{~mL}$ of $50 \mathrm{mM}$ Tris- $\mathrm{HCl}, \mathrm{pH} 7.5,0.2 \mathrm{M} \mathrm{NaCl}, 1.0 \% \mathrm{~N}$-lauryl sarcosine, $1 \%$ Triton X-100 (v/v), $6 \mathrm{M}$ urea. Any material still insoluble was removed by centrifugation at $12000 \mathrm{~g}$ for $10 \mathrm{~min}$ at room temperature.

The solubilized fusion protein was refolded by dialysis against 40 volumes of $50 \mathrm{mM}$ Tris, $\mathrm{pH}$ 7.5, $0.2 \mathrm{M} \mathrm{NaCl}, 1 \%$ Triton X-100 (v/v) for $24 \mathrm{~h}$. Following dialysis, cleavage of the $g y r A$ intein to generate the thioester at the $\mathrm{C}$-terminus of the $\mathrm{N}$-peptide sequence was carried out by the addition of solid 2-mercaptoethanesulfonic acid (MESNA) to $0.1 \mathrm{M}$. Almost complete cleavage of the intein was observed after overnight incubation at room temperature. The cleaved gyrA intein along with any uncleaved fusion proteins were removed by passage through a chitin resin column. The GST-N-peptide fusion protein thioester obtained after passage through the chitin column was proteolysed using thrombin ( $0.5 \mathrm{U}$ per $\mathrm{mg}$ of fusion) to remove GST. The N-peptide thioester that is released by proteolysis was purified using RPHPLC. Triton X-100 used in the purification steps interferes with the RP-HPLC purification and was therefore removed by trichloroacetic acid precipitation and acetone washes as previously described.(15) The N-peptide thioester was purified on a preparative $\mathrm{C} 4$ column using a gradient of $40-90 \%$ buffer B. This protocol yielded $\sim 5 \mathrm{mg}$ of the purified N-peptide thioester, which was characterized as the desired product by ES-MS [observed mass $=5649.1$ \pm 0.5 , expected $=5648.5]$. 


\section{Unfolding and refolding of recombinant NaK channel}

The NaK channel was expressed and purified as previously described.(3) For in vitro folding studies, the NaK channel was unfolded by the addition of trichloroacetic acid to $10 \%(\mathrm{w} / \mathrm{v})$. The mixture was incubated at $4{ }^{\circ} \mathrm{C}$ for $30 \mathrm{~min}$ and the protein precipitate was collected by centrifugation at $3000 \mathrm{~g}$. The protein precipitate was washed with ice cold acetone, dissolved in 50\% TFE: buffer $\mathrm{A}\left(\mathrm{H}_{2} \mathrm{O}, 0.1 \% \mathrm{TFA}\right)$ and then lyophilized. The lyophilized protein was dissolved in $0.1 \mathrm{M}$ sodium phosphate, $\mathrm{pH} 8.0,0.1 \mathrm{M}$ DTT, $1 \%$ SDS. Complete unfolding of the NaK channel was confirmed by SDS-PAGE. For refolding, the unfolded protein was diluted $(1 \rightarrow 10)$ into Soybean phosphatidylcholine vesicles $(20 \mathrm{mg} / \mathrm{mL}$ in $50 \mathrm{mM} 2-$

morpholinoethanesulfonic acid, $\mathrm{pH} 6.4,0.3 \mathrm{M} \mathrm{KCl}, 10 \mathrm{mM}$ DTT). The mixture was sonicated in a water bath sonicator and then incubated at room temperature. Refolding of the $\mathrm{NaK}$ channel was monitored by the appearance of a tetramer band on SDS-PAGE.

\section{Assembly and purification of semisynthetic NaK channel}

The semisynthetic $\mathrm{NaK}$ polypeptide was assembled from the N-peptide thioester and the $\mathrm{C}$ peptide by NCL. We typically used a 2-fold excess of the C-peptide over the N-peptide thioester. Prior to setting up the NCL reaction, the peptides were co-lyophilized with dodecylphosphocholine (Fos-12, final concentration 1\% w/v), which enables easy solubilization of the peptides in the ligation buffer. The ligation reaction was carried out in $0.1 \mathrm{M}$ sodium phosphate, $\mathrm{pH}$ 7.4, $20 \mathrm{mM}$ tris-(2-carboxyethyl)-phosphine (TCEP, hydrochloride salt). Thiophenol was added to $2 \%$ and the reaction was carried out at $37{ }^{\circ} \mathrm{C}$ with gentle stirring. SDS-PAGE was used to monitor the progress of the ligation reaction. The ligation reactions were terminated by the addition of DTT to $0.1 \mathrm{M}$.

Folding reactions for the semisynthetic $\mathrm{NaK}$ channels were carried out on the ligation reaction mixtures itself without further purification. Folding of the semisynthetic polypeptides was carried out as described for the unfolded recombinant protein and was monitored by SDSPAGE.

For purification, the folding reaction mixture was initially dialyzed against $50 \mathrm{mM}$ Tris- $\mathrm{HCl}$, $\mathrm{pH} 7.5,0.3 \mathrm{M} \mathrm{KCl}$ for removal of DTT. The lipid vesicles were solubilized by the addition of $40 \mathrm{mM}$ decylmaltoside (DM) and the proteins were purified from the lipids using immobilized metal affinity chromatography. The folded semisynthetic $\mathrm{NaK}$ channels were then separated from the unfolded (monomeric) proteins and the un-ligated peptides by size exclusion chromatography on a Superdex 200 column using a running buffer consisting of $50 \mathrm{mM}$ Tris$\mathrm{HCl}, \mathrm{pH} 7.5,150 \mathrm{mM} \mathrm{KCl}, 10 \mathrm{mM} \mathrm{DM}, 2 \mathrm{mM}$ DTT. Fractions containing the folded tetrameric $\mathrm{NaK}$ channel were identified by SDS-PAGE, pooled and stored at $-80^{\circ} \mathrm{C}$ until use. Protein identity was confirmed by matrix-assisted laser desorption ionization mass spectrometry (MALDI-MS) that was carried out at the Mass Spectrometry Laboratory, Oregon State University, Corvallis, OR.

\section{${ }^{86} \mathrm{Rb}^{+}$Flux Assay}

For ${ }^{86} \mathrm{Rb}^{+}$flux assays, the semisynthetic NaK channels were reconstituted into 1-palmitoyl-2oleoyl-glycero-3-phosphatidylethanolamine (POPE) /1-palmitoyl-2-oleoyl-glycero-3phosphatidylglycerol (POPG) (3:1) lipid vesicles at $10 \mu \mathrm{g}$ of protein $/ \mathrm{mg}$ of lipid. For reconstitution, the protein was added to lipids solubilized in $10 \mathrm{mM}$ HEPES-KOH, $\mathrm{pH} 7.5$, $450 \mathrm{mM} \mathrm{KCl}, 10 \mathrm{mM} \mathrm{DM}$ and lipid vesicles were formed by removal of detergent using dialysis against $10 \mathrm{mM}$ HEPES-KOH, $\mathrm{pH} 7.4,450 \mathrm{mM} \mathrm{KCl}$. The ${ }^{86} \mathrm{Rb}^{+}$flux assays were carried out as previously described. $(3,16)$ The readings obtained in the flux assays were normalized to the level of equilibrium ${ }^{86} \mathrm{Rb}^{+}$uptake into all the lipid vesicles present. For this purpose, valinomycin was added to the reaction mixture at a final concentration of $1 \mu \mathrm{g} / \mathrm{mL}$ and an aliquot measured for ${ }^{86} \mathrm{Rb}^{+}$uptake after further incubation for $1-2 \mathrm{~min}$. For the measurement 
of $\mathrm{Ca}^{2+}$ block of the ${ }^{86} \mathrm{Rb}^{+}$flux, $\mathrm{Ca}^{2+}$ was directly into the flux buffer at the desired final concentrations. Ion flux was allowed to proceed for $10 \mathrm{~min}$ and ${ }^{86} \mathrm{Rb}^{+}$uptake was measured. (4)

\section{Results and Discussion}

\section{Synthetic strategy}

Our strategy for the synthesis of the NaK channel consists of two steps; the assembly of the $\mathrm{NaK}$ polypeptide and the in vitro folding of the $\mathrm{NaK}$ polypeptide to the native tetrameric state (Fig. 1C). Each subunit of the NaK channel is 110 amino acids long, which is beyond the limits of SPPS.(9) SPPS is only efficient for the synthesis of peptides 50-60 amino acids in length. (9) The NaK subunit is therefore assembled from two component peptides by NCL.(17) In NCL, a peptide with an N-terminal Cys (N-Cys) reacts with a $\alpha$-thioester peptide to link the two peptides with a native peptide bond at the ligation site. Leu54 was selected as the ligation site as biochemical analysis indicated that a Cys substitution at this position, which is required for the NCL reaction, is well tolerated (data not shown). The Leu54 ligation site is advantageous as the residues that form the ion binding sites (Thr63-Gly67, the region of interest in the $\mathrm{NaK}$ channel) are C-terminal to the ligation site (Fig. 1C). This allowed us to pursue a semisynthetic approach in which the protein segment $\mathrm{C}$-terminal to the ligation site that contains the region of interest was obtained by chemical synthesis while the protein segment $\mathrm{N}$-terminal to the ligation site was obtained by recombinant means. Prior analysis of the $\mathrm{NaK}$ channel has indicated that the deletion of residues 1-19 at the N-terminus and substitution of Phe92 with Ala increases channel activity in ${ }^{86} \mathrm{Rb}^{+}$flux assays. $(3,18)$ These modifications were therefore incorporated into our semisynthetic construct for ease of functional characterization. The semisynthesis of the $\mathrm{NaK}$ channel therefore calls for the generation of a recombinant $\mathrm{N}$ terminal peptide thioester corresponding to residues 19-53 (N-peptide) and a synthetic Cterminal peptide with a N-Cys corresponding to residues 54-110 (C-peptide).

\section{Synthesis and purification of the C-peptide}

The 57 amino acid C-peptide was synthesized by manual SPPS using Boc protection and in situ neutralization/HBTU activation.(13) Following synthesis, global de-protection and cleavage from the resin was carried out using anhydrous HF. The peptide crude obtained after HF cleavage was soluble in 50\% TFE: Buffer A. ES-MS of the peptide crude showed the presence of the desired peptide indicating a successful synthesis. However, purification of the C-peptide was not possible as the peptide crude could not be resolved using RP-HPLC (Fig 2A). An inspection of the sequence of the C-peptide suggested that the difficulties in purification might arise from a paucity of positively charged residues.(19) To circumvent this problem, we designed a purification strategy for the C-peptide that involves a temporary introduction of positively charged residues. Our strategy was based on previous reports of the temporary introduction of Arg residues for the purification of membrane spanning thioester fragments required for the chemical synthesis of membrane proteins. In these cases, 5-6 Arg residues were appended to the peptide through the thioester linkage. $(20,21)$ The C-peptide is not a thioester peptide but an N-Cys peptide therefore a thioester linkage could not be used for appending the Arg residues. Instead, the thiol side chain of the N-Cys was used as the site of attachment and the Arg residues were linked to the side chain through a disulfide bond.

Following purification, the Arg residues can be released from the C-peptide by cleaving the disulfide linkage by reducing agents ad therefore the Arg residues that are temporarily introduced to enable purification are not present in the final product, the semisynthetic $\mathrm{NaK}$ channel.

To test this strategy, we synthesized a temporary solubilizing tag peptide (TST) with the sequence RRRC. To modify the C-peptide with the TST, we incubated the C-peptide crude 
with the TST in 50\% TFE at pH 8.0. RP-HPLC analysis showed the presence of a new peak which was identified by ES-MS as the C-peptide linked to TST (Fig. 2B). Using the TST strategy, we obtained $\sim 2 \mathrm{mg}$ of the purified C-peptide from $90 \mathrm{mg}$ of the peptide crude (Fig. $2 \mathrm{C}, \mathrm{D})$. The TST strategy used for the purification of the C-peptide should have general applicability in the purification of membrane spanning N-Cys peptides segments required for the synthesis of integral membrane proteins.

\section{Recombinant expression of the N-peptide thioester}

To introduce the $\alpha$-thioester group, the N-peptide sequence was fused to the gyrA intein.(22) We used a dual-fusion approach for the expression of the $\mathrm{N}$-peptide-intein fusion as expression of membrane spanning peptides fused to an intein results in cell lethality (Fig. 3A).(14) In the dual-fusion approach, GST is appended to the N-terminus of the NaK-intein fusion. The Nterminal GST directs expression of the fusion protein to inclusion bodies thereby avoiding cell lethality.(23) Following expression as inclusion bodies, the dual-fusion protein was purified, solubilized using urea and then folded in vitro. The gyrA intein was cleaved using MESNA to generate the GST-N-peptide thioester. GST was then removed by proteolysis to generate the N-peptide thioester, which was purified by RP-HPLC and confirmed using ES-MS (Fig. 3B). Using this procedure, we obtained $\sim 5 \mathrm{mg}$ of the $\mathrm{N}$-peptide thioester per $\mathrm{L}$ of bacterial culture.

\section{Assembly of semi-synthetic NaK channels}

The NaK polypeptide was assembled from the N-peptide thioester and the synthetic C-peptide using the NCL reaction. The NCL reaction was carried out in the presence of detergents, which were required to keep the peptides soluble during the course of the reaction. A survey of detergents indicated that the NCL reaction proceeded readily when the peptides were solubilized in Fos-12. Prior to the NCL reaction, we unmasked the Cys residue at the Nterminus of the C-peptide by cleaving the disulfide bond to the TST peptide using $10 \mathrm{mM}$ TCEP. The ligation reaction was initiated by the addition of thiophenol to $2 \%$ and monitored by SDS-PAGE. The ligation reaction was $\sim 80 \%$ complete after 24 hour (Fig. 4A). MALDIMS of the ligated material indicated that it has a mass consistent with the expected product [observed mass $=11600.6$, expected $=11600$ ] $($ Fig. 4B $)$.

A key requirement of the semisynthesis is the in vitro folding of the $\mathrm{NaK}$ polypeptide to the native state. As in vitro folding of the $\mathrm{NaK}$ channel had not been previously reported, we initially investigated conditions for in vitro folding of the $\mathrm{NaK}$ channel. These experiments were carried out using the unfolded recombinant protein. The $\mathrm{NaK}$ channel can be unfolded by heating in the presence of SDS or by precipitation with acid/organic solvents. The native $\mathrm{NaK}$ channel migrates as a partial tetramer on SDS-PAGE while the unfolded $\mathrm{NaK}$ polypeptide migrates as a monomer (Fig. 5A). SDS-PAGE, therefore provides an easy means to determine the folded state of the $\mathrm{NaK}$ channel. Our analysis indicated that refolding of the NaK channel was observed on incorporation of the unfolded polypeptide into lipid vesicles. The refolding of the NaK channel was indicated by the appearance of a tetrameric band on SDS-PAGE of the folding reaction (Fig. 5A).

Folding of the semisynthetic $\mathrm{NaK}$ polypeptide was carried out on the ligation reaction mixture itself without further purification. For folding, the ligation reaction mixture was diluted into lipid vesicles. Folding of the semisynthetic $\mathrm{NaK}$ channel was detected by the appearance of a tetrameric band on SDS-PAGE (Fig. 5B). The semisynthetic NaK channels carry a His ${ }_{6}$ tag at the N-terminus, which facilitated a two step purification of the folded channels. An initial metal affinity chromatography step was used to separate the folded and unfolded $\mathrm{NaK}$ channels from the lipid vesicles, which was followed by a size exclusion chromatography step to separate the folded $\mathrm{NaK}$ channels from the unfolded molecules and the un-reacted peptides. In a typical 
semisynthesis, $0.3 \mathrm{mg}$ of the folded semisynthetic $\mathrm{NaK}$ channel was obtained starting with 1.5 $\mathrm{mg}$ of the $\mathrm{N}$-peptide thioester and $3 \mathrm{mg}$ of the $\mathrm{C}$-peptide.

The semisynthetic $\mathrm{NaK}$ channels were incorporated into lipid vesicles for functional characterization. Activity of the $\mathrm{NaK}$ channel was determined using ${ }^{86} \mathrm{Rb}^{+}$flux assays.(3) Robust uptake of ${ }^{86} \mathrm{Rb}^{+}$was observed for lipid vesicles containing the semisynthetic NaK channels indicating that the semisynthetic channels were functional (Fig. 5C). The NaK channels also conduct $\mathrm{Ca}^{2+}$ ions but do so at a slower rate than $\mathrm{Rb}^{+}$. $\mathrm{Ca}^{2+}$ therefore acts act as a blocker of ${ }^{86} \mathrm{Rb}^{+}$flux through the NaK channel. $(4,5)$ The semisynthetic NaK channels showed similar levels of $\mathrm{Ca}^{2+}$ block of ${ }^{86} \mathrm{Rb}^{+}$flux compared to the recombinant wild type channel (Fig. 5D). These results indicate the functional similarity of the semisynthetic NaK channels to the wild type channels.

\section{Substitutions of Asp66 using semisynthesis}

The NaK channel has an acidic residue, Asp66, in the selectivity filter instead of the highly conserved aromatic residue (Tyr or Phe) that is observed in the selectivity filter of $\mathrm{K}^{+}$channels (Fig. 1B).(5) To investigate the role of Asp66 in NaK channel function, we used semisynthesis to substitute Asp66 with the unnatural amino acids, homoserine (Hse) and cysteine sulfonic acid (Csa). These unnatural amino acids are structurally similar to Asp except that Hse side chain is neutral while the Csa side chain is charged (Fig. 6A). These unnatural amino acid substitutions therefore test the requirement of a negative charge on residue 66 for channel function.

To assemble the mutant $\mathrm{NaK}$ channels, we synthesized C-peptides carrying the desired substitutions. The C-peptides were purified using TST strategy and ligated to the N-peptide thioester to generate the Asp66 substituted NaK polypeptides. MALDI-MS of the ligated materials indicated masses consistent with the expected products [For NaK(Asp66 $\rightarrow$ Hse), observed mass $=11587.2$, expected $=11586.4$; for $\mathrm{NaK}($ Asp66 $\rightarrow$ Csa $)$, observed mass $=$ 11637.7, expected $=11636.4$ ]. We observed similar levels of in vitro folding for the Hse and the Csa mutants, compared to the wild type indicating that both the substitutions were structurally well tolerated. The Hse and Csa mutants were purified by an initial metal affinity chromatography step to separate the folded and unfolded NaK channels from the lipid vesicles, followed by a size exclusion chromatography step to separate the folded channels from the unfolded molecules and the un-reacted peptides. For functional comparison, the folded Hse and the Csa mutant channels were reconstituted into lipid vesicles at a protein to lipid ratio of 1:100. The effect of the Asp66 substitutions on channel activity was tested using ${ }^{86} \mathrm{Rb}^{+}$flux assays (Fig. 6B). Flux activity was observed for both the Hse and the Csa mutants albeit at different levels; low levels of ${ }^{86} \mathrm{Rb}^{+}$flux was observed for the Hse mutant while flux activity slightly lower than the wild type protein was observed for the Csa mutant. The Hse mutant and the Csa mutant showed similar levels of $\mathrm{Ca}^{2+}$ block of ${ }^{86} \mathrm{Rb}^{+}$flux compared to the wild type protein, which suggests that the Hse and the Csa substitutions do not significantly alter the structure of the selectivity filter (Fig. 6C). The reduction in the ${ }^{86} \mathrm{Rb}^{+}$flux activity of the Hse mutant could arise either due to a reduction in the single channel conductance or due to a reduction in the channel open probability. We cannot differentiate between these possibilities using the ${ }^{86} \mathrm{Rb}^{+}$flux assays and therefore we cannot attribute a specific role for the negative charge on residue 66 on $\mathrm{NaK}$ channel function. Our results however do indicate that the negative charge on residue 66 is required for optimal ion flux through the $\mathrm{NaK}$ channel.

\section{Conclusion}

We have developed a semisynthesis for the non-selective cation channel, NaK. In the semisynthesis, the $\mathrm{NaK}$ polypeptide is assembled from a recombinantly expressed peptide thioester and a chemically synthesized N-Cys peptide using the NCL reaction. We have 
developed a purification strategy for the N-Cys peptide in which N-Cys peptide is temporarily tagged with Arg residues to enable purification. We demonstrate the efficient ligation of the recombinant peptide thioester and the chemically synthesized $\mathrm{N}$-Cys peptide to form the semisynthetic polypeptide. We demonstrate that the semisynthetic NaK polypeptide can be folded in vitro using lipid vesicles. Characterization of the semisynthetic NaK channels indicates that they are functionally similar to the recombinant channels. As the protein segments that form the ion binding sites are contained within the chemically synthesized peptide, the semisynthesis enables the use of chemical synthesis to investigate the ion binding sites in the $\mathrm{NaK}$ channel. We demonstrate the facile introduction of unnatural amino acids into the $\mathrm{NaK}$ channel by substituting Asp66 in the selectivity filter region with unnatural amino acids. Our functional analysis indicates that the negative charge of the Asp66 residue is important for optimal flux of ions through the $\mathrm{NaK}$ channel.

A limitation of the current approach is the yield of the semisynthetic NaK channel obtained. The yield obtained using the current approach is sufficient for functional studies but it is not sufficient for structural studies on the semisynthetic channels using x-ray crystallography. The limiting step in the current approach is the purification of the C-peptide. We envision two approaches to improving the yields of the C-peptide. The first approach would be to optimize the TST, for example, by increasing the number of positively charges in the TST. The alternate approach would be to assemble the C-peptide from two component peptides using NCL. In this approach, we expect that the shorter size of the component peptides would lead to easier purification and thereby higher yields of the C-peptide. We also anticipate that the three segment approach would be beneficial in the incorporation of synthetically challenging substitutions (for example, peptide backbone substitutions) into the NaK channel. We are presently investigating these approaches.

In conclusion, we have developed a semisynthesis for the NaK channel. We anticipate that the procedures used in this semisynthesis will also find application in the semisynthesis of other membrane proteins of interest.

\section{Acknowledgments}

We thank Drs. Jeffrey Karpen and Alexander Komarov for critical comments on the manuscript.

The research was supported by grants to FIV from the NIH (GM087546) and a Pew Scholar Award. MGD is supported by a NIH NRSA Pre-doctoral fellowship (GM07703). YJ is an investigator in the Howard Hughes Medical Institute.

\section{Abbreviations}

$\begin{array}{ll}\text { NCL } & \text { native chemical ligation } \\ \text { SPPS } & \text { solid phase peptide synthesis } \\ \text { HBTU } & \text { 2-(1H-benzo-triazol-1-yl)-1,1,3,3-tetramethyluronium hexafluorophosphate } \\ \text { DMSO } & \text { dimethyl sulfoxide } \\ \text { HF } & \text { hydrogen fluoride } \\ \text { TFE } & \text { trifluoroethanol } \\ \text { TFA } & \text { trifluoroacetic acid } \\ \text { TST } & \text { temporary solubilizing tag } \\ \text { MBHA } & \text { 4-methylbenzylhydrylamine } \\ \text { ES-MS } & \text { electrospray-mass spectrometry }\end{array}$




$\begin{array}{ll}\text { Hse } & \text { homoserine } \\ \text { Csa } & \text { cysteine sulfonic acid } \\ \text { GST } & \text { glutathione-S-transferase } \\ \text { MESNA } & \text { 2-mercaptoethane sulfonic acid } \\ \text { DM } & \text { decyl maltoside } \\ \text { Fos-12 } & \text { dodecyl phosphocholine } \\ \text { TCEP } & \text { tris-(2-carboxyethyl)-phosphine } \\ \text { MALDI-MS } & \text { matrix-assisted laser desorption ionization mass spectrometry }\end{array}$

\section{References}

1. Hille, B. Ion Channels of Excitable Membranes. Third ed.. Sunderland, MA: Sinauer Associates, Inc; 2001. p. 2-22.

2. Craven KB, Zagotta WN. CNG and HCN channels: two peas, one pod. Annu. Rev. Physiol 2006;68:375-401. [PubMed: 16460277]

3. Shi N, Ye S, Alam A, Chen LP, Jiang Y. Atomic structure of a Na+- and K+-conducting channel. Nature 2006;440:570-574. [PubMed: 16467789]

4. Alam A, Jiang Y. Structural analysis of ion selectivity in the NaK channel. Nat. Struct. Mol. Biol 2009;16:35-41. [PubMed: 19098915]

5. Alam A, Shi N, Jiang Y. Structural insight into Ca2+ specificity in tetrameric cation channels. Proc. Natl. Acad. Sci. U S A 2007;104:15334-15339. [PubMed: 17878296]

6. Zhou Y, Morais-Cabral J, Kaufman A, MacKinnon R. Chemistry of ion coordination and hydration revealed by a K+ channel-Fab complex at 2.0 A resolution. Nature 2001;414:43-48. [PubMed: 11689936]

7. Jiang Y, Lee A, Chen J, Ruta V, Cadene M, Chait B, MacKinnon R. X-ray structure of a voltagedependent K+ channel. Nature 2003;423:33-41. [PubMed: 12721618]

8. Long SB, Tao X, Campbell EB, MacKinnon R. Atomic structure of a voltage-dependent $\mathrm{K}+$ channel in a lipid membrane-like environment. Nature 2007;450:376-382. [PubMed: 18004376]

9. Kent S. Chemical synthesis of peptides and proteins. Annu. Rev. Biochem 1988;57:957-989. [PubMed: 3052294]

10. Olschewski D, Becker CF. Chemical synthesis and semisynthesis of membrane proteins. Mol. Biosyst 2008;4:733-740. [PubMed: 18563247]

11. Johnson ECB, Kent SBH. Studies on the insolubility of a transmembrane peptide from signal peptide peptidase. J. Am. Chem. Soc 2006;128:7140-7141. [PubMed: 16734449]

12. Stanley AM, Fleming KG. The process of folding proteins into membranes: challenges and progress. Arch. Biochem. Biophys 2008;469:46-66. [PubMed: 17971290]

13. Schnolzer M, Alewood P, Jones A, Alewood D, Kent SB. In situ neutralization in Boc-chemistry solid phase peptide synthesis. Rapid, high yield assembly of difficult sequences. Int. J. Pept. Protein Res 1992;40:180-193. [PubMed: 1478777]

14. Valiyaveetil F, MacKinnon R, Muir T. Semisynthesis and folding of the potassium channel KcsA. J. Am. Chem. Soc 2002;124:9113-9120. [PubMed: 12149015]

15. Valiyaveetil FI, Sekedat M, Muir TW, MacKinnon R. Semisynthesis of a functional K+ channel. Angew. Chem., Int. Ed 2004;43:2504-2507.

16. Heginbotham L, Kolmakova-Partensky L, Miller C. Functional reconstitution of a prokaryotic K+ channel. J. Gen. Physiol 1998;111:741-749. [PubMed: 9607934]

17. Dawson PE, Muir TW, Clark Lewis I, Kent SBH. Synthesis of proteins by native chemical ligation. Science 1994;266:776-779. [PubMed: 7973629]

18. Alam A, Jiang Y. High-resolution structure of the open NaK channel. Nat. Struct. Mol. Biol 2009;16:30-34. [PubMed: 19098917] 
19. Melnyk RA, Partridge AW, Yip J, Wu Y, Goto NK, Deber CM. Polar residue tagging of transmembrane peptides. Biopolymers 2003;71:675-685. [PubMed: 14991677]

20. Johnson E, Kent S. Towards the total chemical synthesis of integral membrane proteins: a general method for the synthesis of hydrophobic peptidethioester building blocks. Tetrahedron Lett 2007;48:1795-1799. [PubMed: 19177172]

21. Sato T, Saito Y, Aimoto S. Synthesis of the C-terminal region of opioid receptor like 1 in an SDS micelle by the native chemical ligation: effect of thiol additive and SDS concentration on ligation efficiency. J. Pept. Sci 2005;11:410-416. [PubMed: 15635671]

22. Southworth MW, Amaya K, Evans TC, Xu MQ, Perler FB. Purification of proteins fused to either the amino or carboxy terminus of the Mycobacterium xenopi gyrase A intein. Biotechniques 1999;1999:110-114. [PubMed: 10407673]

23. Frangioni JV, Neel BG. Solubilization and Purification of Enzymatically Active Glutathione-STransferase (pGEX) Fusion Proteins. Anal. Biochem 1993;210:179-187. [PubMed: 8489015] 
A

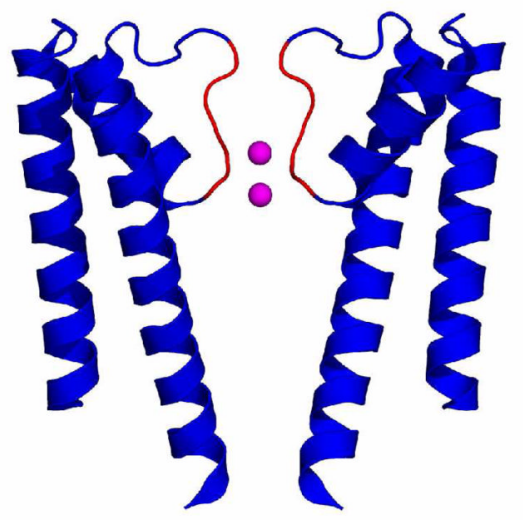

C

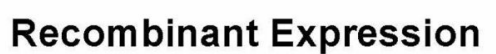

Recombinant Expression
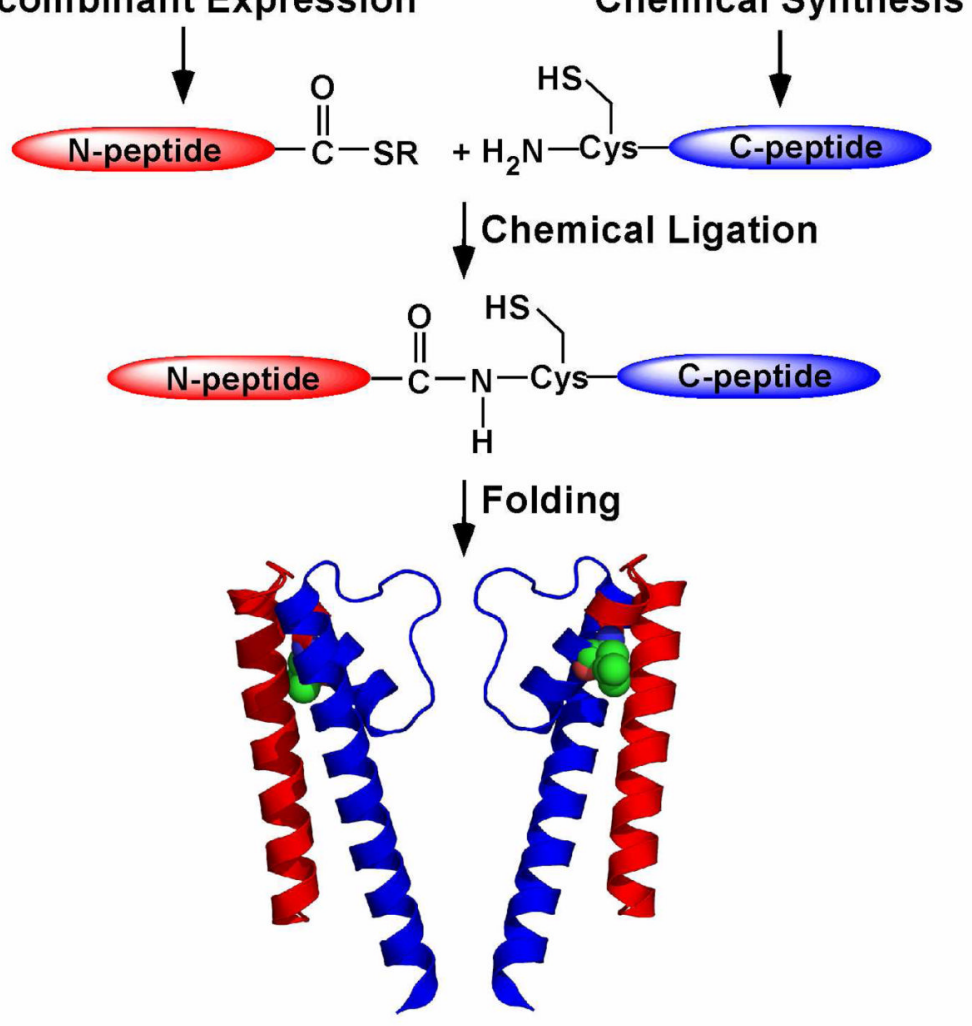

Figure 1. Semisynthesis of NaK

A) Structure of NaK. Two opposite subunits of the tetrameric NaK channel are shown (pdb: 2ahy). The selectivity filter sequence, residues 63-67, is colored red. B) Close-up view of the selectivity filter. Residues $63-67$ are depicted in stick representation. $\mathrm{Na}^{+}$ions bound to the filter sites are depicted as magenta spheres. C) Strategy for the semisynthesis of the $\mathrm{NaK}$ channel. The $\mathrm{NaK}$ polypeptide was synthesized by the native chemical ligation of a recombinant N-peptide thioester (residues 19-53, red) and a synthetic C-peptide (residues 54110 , blue). The NaK polypeptide (19-110) obtained by the ligation reaction is folded to the native tetrameric state. Two subunits of the $\mathrm{NaK}$ tetramer are shown and the ligation site, residue 54 is depicted in space-fill. 

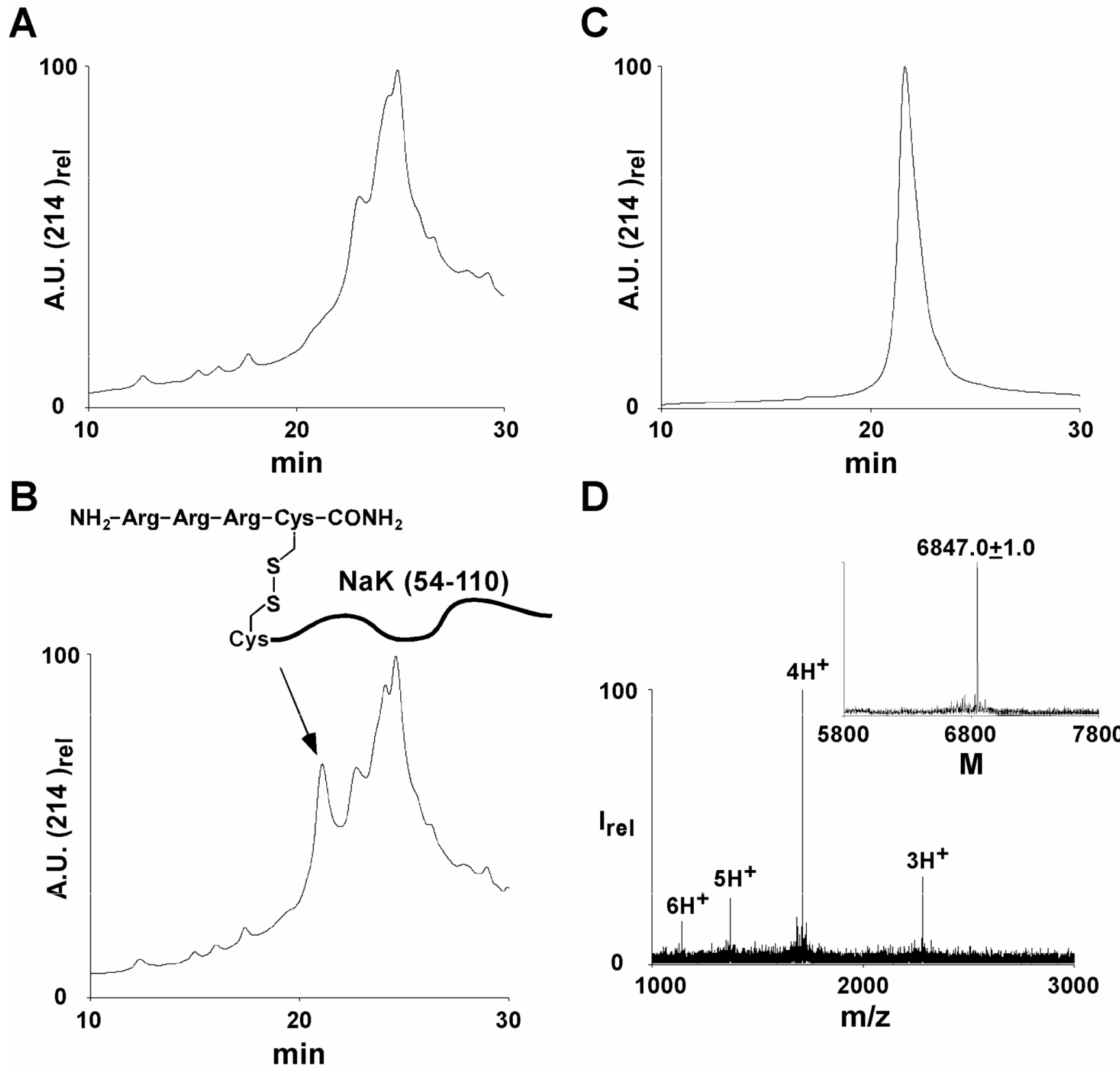

Figure 2. Purification of the C-peptide

A) RP-HPLC of the C-peptide crude. B) RP-HPLC of the C-peptide crude after modification with the temporary solubilizing tag (TST). The peak corresponding to the TST modified Cpeptide is indicated. C) RP-HPLC of the purified TST-C-peptide. D) ES-MS of the purified TST-C-peptide (inset, reconstructed spectrum, expected mass $=6847.8$ ). In all the panels, RPHPLC analysis is carried out on a C4 column using a gradient of 60-100\% Buffer C. 

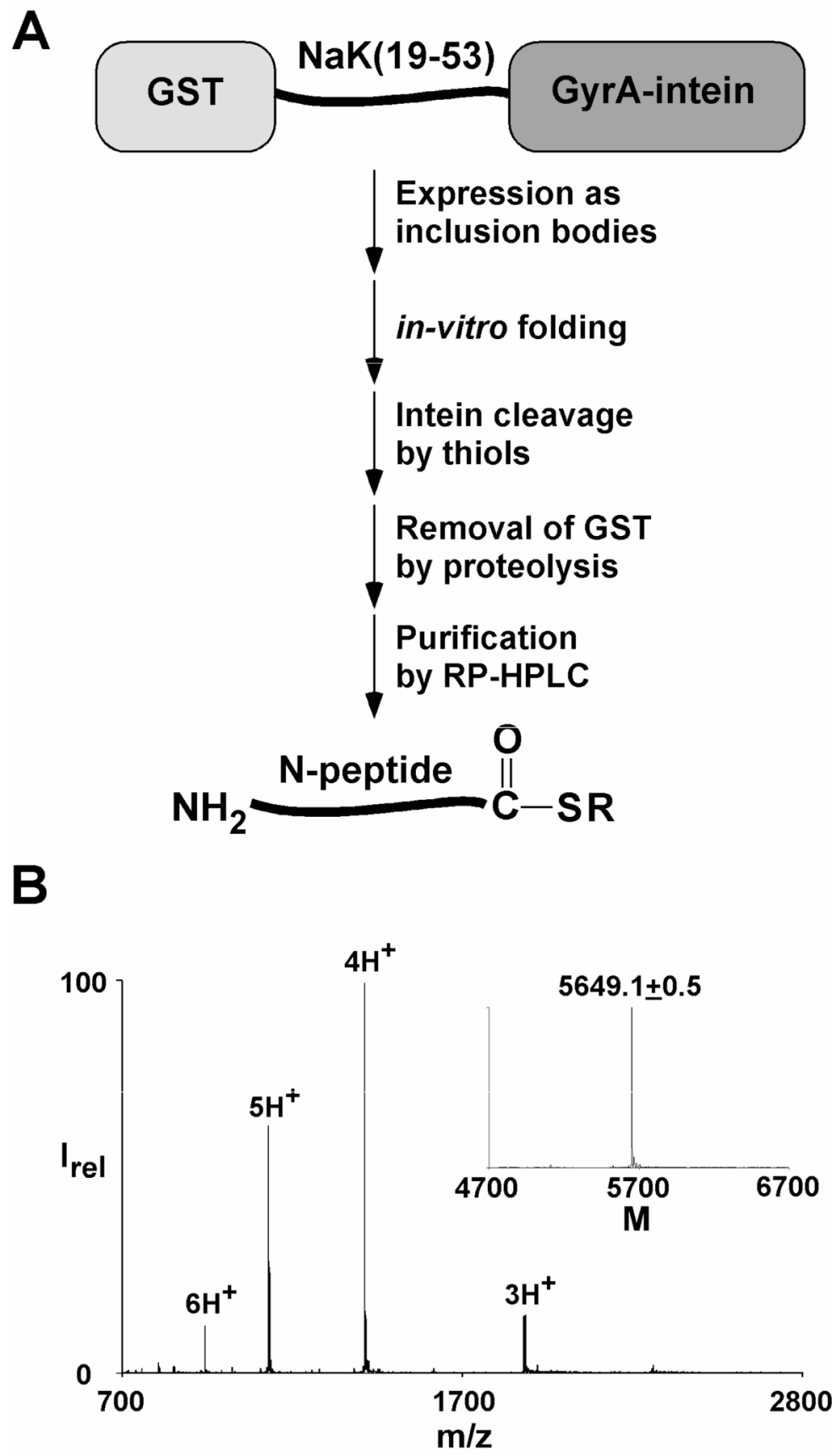

Figure 3. Purification of the N-peptide thioester A) The dual fusion strategy used for the recombinant expression and purification of the Npeptide thioester. B) ES-MS of the purified N-peptide thioester (inset, reconstructed spectrum, expected mass $=5648.5$ ) 


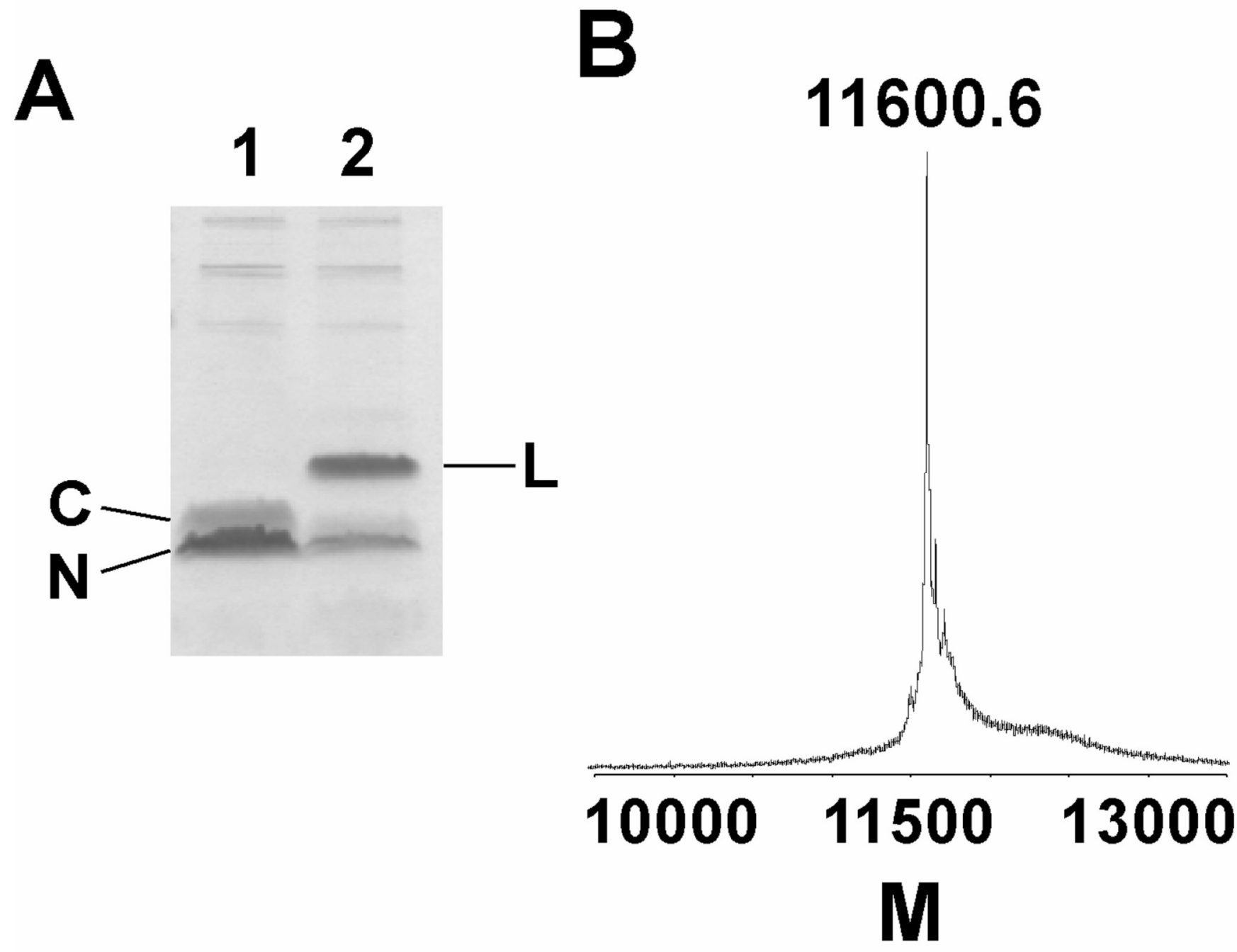

Figure 4. Assembly of NaK by native chemical ligation

A) SDS-PAGE gel of the native chemical ligation reaction at $0 \mathrm{~min}$ (lane 1) and $24 \mathrm{~h}$ (lane 2) showing the C-peptide $(\mathrm{C}), \mathrm{N}$-peptide $(\mathrm{N})$ and the ligation product $(\mathrm{L})$. The faint bands observed, which are higher than the ligation product, correspond to aggregates of the $\mathrm{N}$ or the C-peptide. B) MALDI-MS of the ligation product (expected mass $=11600)$. 
A

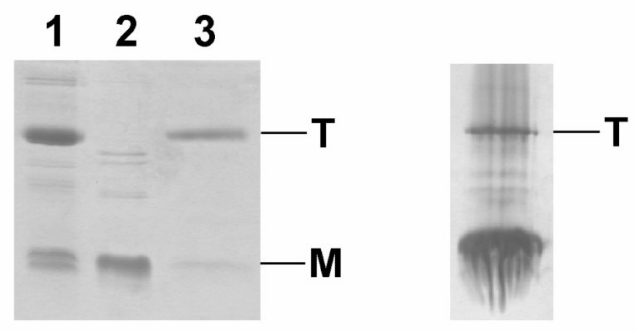

C
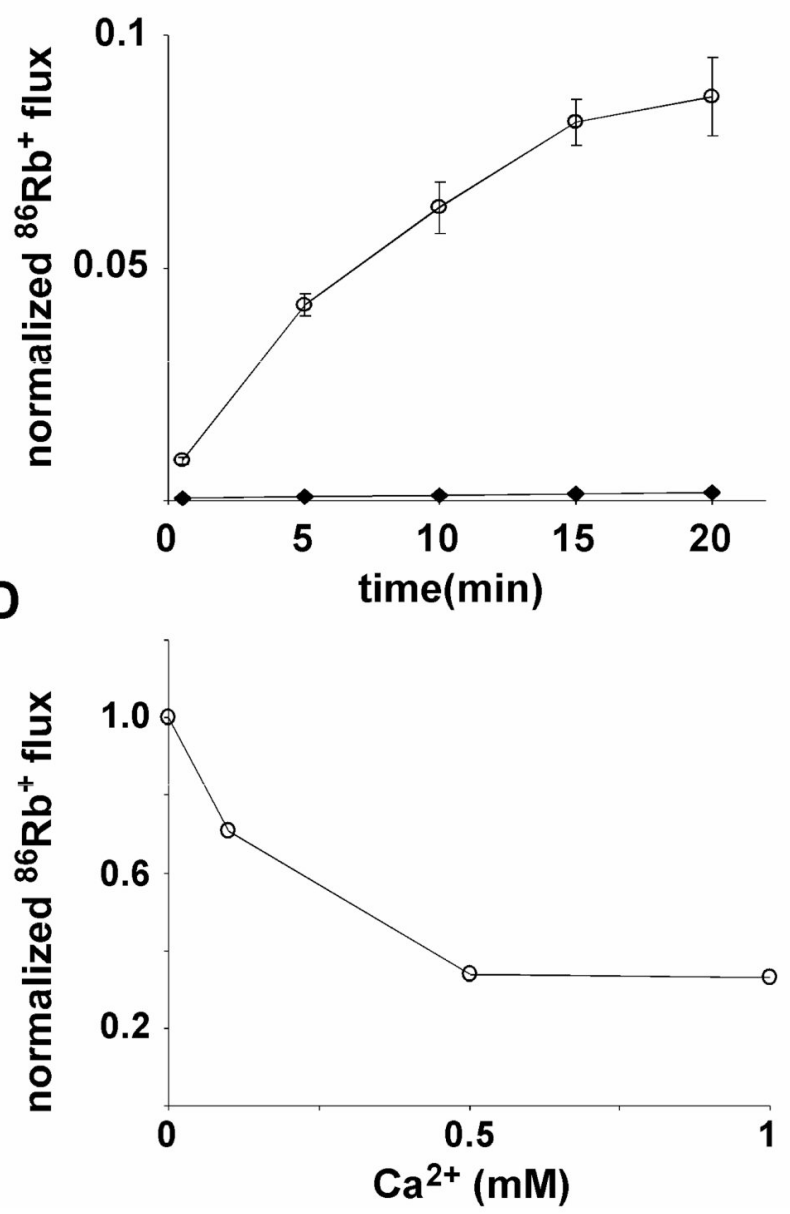

Figure 5. In vitro folding of the NaK channel

A) SDS-PAGE gel showing the unfolding and refolding of the recombinant NaK channel. Native recombinant $\mathrm{NaK}$ (Lane 1), unfolded NaK (Lane 2), refolded NaK channel (Lane 3). B) SDS-PAGE gel showing the folding of semisynthetic NaK channel by lipids. In panels A and $\mathrm{B}$, the folded tetrameric $\mathrm{NaK}$ channel $(\mathrm{T})$ and the unfolded monomeric $\mathrm{NaK}$ polypeptide (M) are indicated. C) ${ }^{86} \mathrm{Rb}^{+}$flux assay for the semisynthetic $\mathrm{NaK}$ channel function. The folded semisynthetic NaK channels were purified by metal affinity chromatography followed by size exclusion chromatography and reconstituted into lipid vesicles. Time course for ${ }^{86} \mathrm{Rb}^{+}$uptake into lipid vesicles loaded with $450 \mathrm{mM} \mathrm{KCl}$ reconstituted with semisynthetic $\mathrm{NaK}$ channel (o) and the control lipid vesicles without protein $(\diamond)$. The observed ${ }^{86} \mathrm{Rb}^{+}$uptake was normalized 
to extent of valinomycin-induced uptake in the same vesicles. D) $\mathrm{Ca}^{2+}$ block of $\mathrm{Rb}^{+}$flux into lipid vesicles reconstituted with the semisynthetic NaK channel. 
A

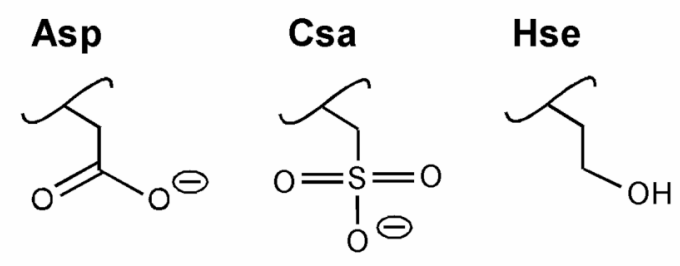
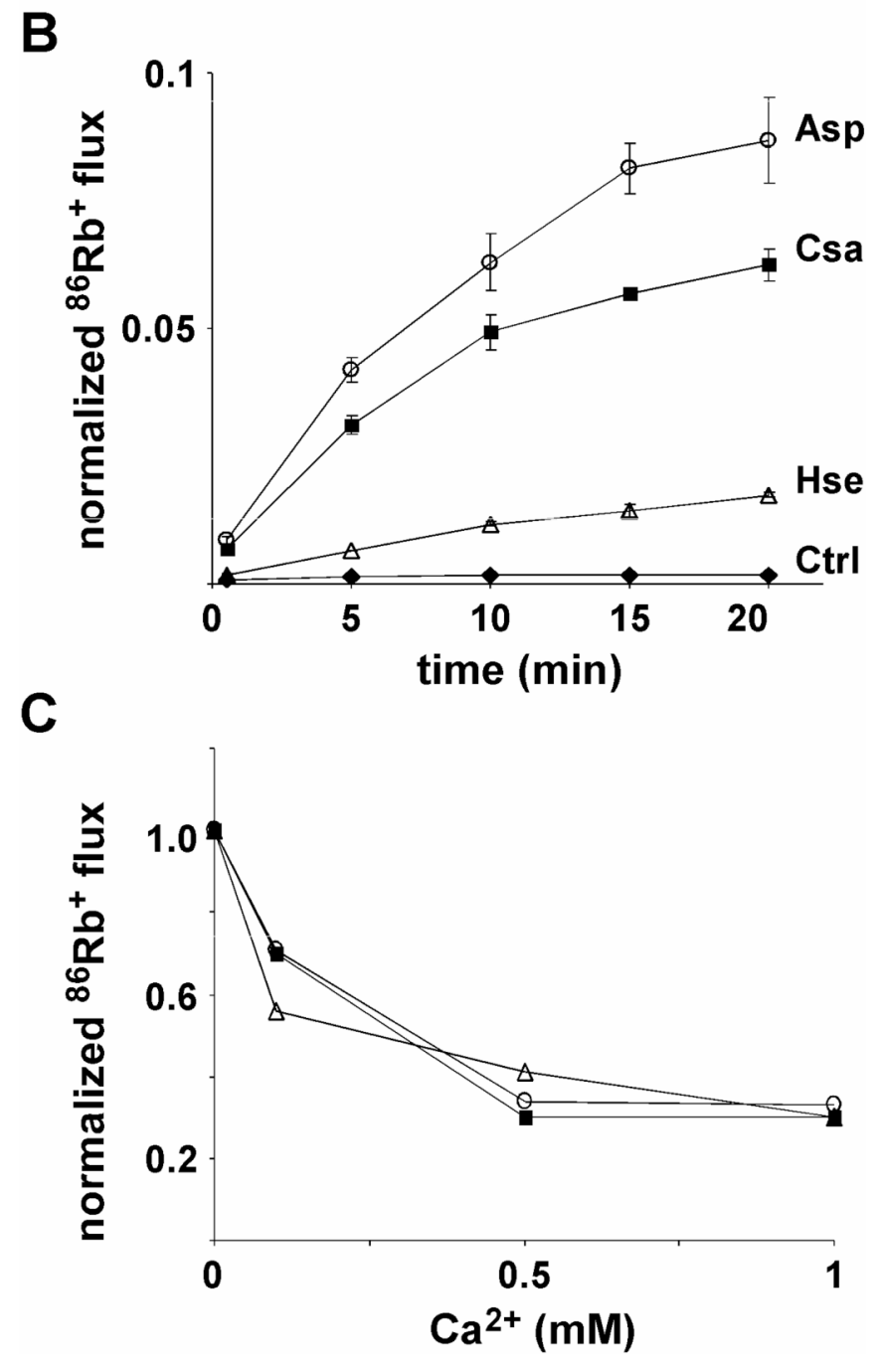

Figure 6. Asp66 mutants of the NaK channel

A) Structures of the unnatural amino acids, cysteine sulfonic acid (Csa) and homoserine (Hse). B) Time course for ${ }^{86} \mathrm{Rb}^{+}$uptake into lipid vesicles loaded with $450 \mathrm{mM} \mathrm{KCl}$ reconstituted with the semisynthetic NaK channels and the control lipid vesicles without protein $(\downarrow)$. The observed ${ }^{86} \mathrm{Rb}^{+}$uptake was normalized to extent of valinomycin-induced uptake in the same vesicles. C) $\mathrm{Ca}^{2+}$ block of $\mathrm{Rb}^{+}$flux into lipid vesicles reconstituted with the semisynthetic $\mathrm{NaK}$ channels. For panels $\mathbf{B}$ and $\mathbf{C}$, wild type (Asp66) semisynthetic NaK channel (O), the Asp66 $\rightarrow$ Csa mutant $(\mathbf{a})$ and the Asp66 $\rightarrow$ Hse mutant $(\Delta)$. 